Article

\title{
An Optimized and Sensitive Pharmacokinetic Quantitative Method of Investigating Gastrodin, Parishin, and Parishin B, C and E in Beagle Dog Plasma using LC-MS/MS after Intragastric Administration of Tall Gastrodia Capsules
}

\author{
Junqiu Liu ${ }^{\dagger}$, Sha Chen ${ }^{+} \mathbb{D}^{\circ}$, Jintang Cheng, Jun Zhang, Yuesheng Wang and An Liu * \\ Key laboratory of Beijing for identification and safety evaluation of Chinese medicine, \\ Institute of Chinese Materia Medica, China Academy of Chinese Medical Sciences, No. 16, Nanxiaojie, \\ Dongzhimennei, Beijing 100700, China; liujoal@163.com (J.L.); schen@icmm.ac.cn (S.C.); \\ jtcheng@icmm.ac.cn (J.C.); jzhang@icmm.ac.cn (J.Z.); yswang@icmm.ac.cn (Y.W.) \\ * Correspondence: aliu@icmm.ac.cn; Tel.: +86-010-6401-4411 \\ + These authors contributed equally to this paper.
}

Received: 10 October 2017; Accepted: 7 November 2017; Published: 10 November 2017

\begin{abstract}
Gastrodia elata Blume, called Tianma in China, has been widely used to treat headaches, convulsions and epilepsy for thousands of years. In the present study, a series of optimizations were employed to develop a rapid, sensitive, and reliable high-performance liquid chromatography-triple quadrupole mass spectrometry method, which was then used for the simultaneous determination of gastrodin, parishin, parishin B, parishin $\mathrm{C}$ and parishin $\mathrm{E}$ in beagle dog plasma after intragastric administration of tall Gastrodia capsules (Tianma brand). The chromatographic separation was achieved on a $\mathrm{C}_{18}$ column with gradient elution by using a mixture of $0.4 \%$ formic acid aqueous solution and acetonitrile as the mobile phase at a flow rate of $0.15 \mathrm{~mL} / \mathrm{min}$. A tandem mass spectrometric detection was conducted using multiple-reaction monitoring (MRM) via electrospray ionization (ESI) source in negative ionization mode. Samples were pre-treated by a single-step protein precipitation with methanol, and bergenin was used as internal standard (IS). Under the optimized conditions, the lower limit of quantification (LLOQ) was $0.10 \mathrm{ng} / \mathrm{mL}$ for gastrodin, $0.40 \mathrm{ng} / \mathrm{mL}$ for parishin $\mathrm{B}, 0.02 \mathrm{ng} / \mathrm{mL}$ for parishin $\mathrm{E}$ and $0.20 \mathrm{ng} / \mathrm{mL}$ for parishin and parishin $\mathrm{C}$, all of which previously were the highest levels of sensitivity. The methods were optimized for selectivity, calibration curves, accuracy and precision. Extraction recoveries, matrix effects and stability were within acceptable ranges. Pharmacokinetic parameters of the tested substances were also quantitatively determined. Finally, a possible metabolic pathway was induced based on correlations obtained from quantitative and qualitative data analysis in vivo.
\end{abstract}

Keywords: gastrodin; parishin; tall Gastrodia capsules; pharmacokinetics; correlation; LC-MS/MS

\section{Introduction}

Pharmacokinetic studies on Gastrodia elata Blume (GE, tianma) have mainly focused on gastrodin and one or two parishin and hydroxybenzyl alcohol compounds in dogs and rats [1-3]. To date, pharmacokinetic research on compounds like gastrodin, $p$-hydroxybenzyl alcohol and parishin compounds has typically been conducted in rodents. Some bottlenecks exist however in studying the oral administration pharmacokinetics of these compounds in plasma, such as a lack of a highly sensitive method of integrated analysis of gastrodin and parishins, or a clear method for explaining the inner links among these bioactive compounds in vivo. In addition to the high quantitative limit 
and transformation of compounds, suitable acidity of the mobile phase is also key for bio-availability analysis in vivo for GE compounds analysis. However, the mobile phase acidity can change the charged condition of ions, also influencing the sensitivity of quantitative methods. Thus, there is an urgent need to establish a systematic analysis protocol.

Research on GE has recently focused primarily on metabolism. It was shown that in vivo, parishin can be biotransformed into a variety of other metabolites including parishin B, parishin E, and parishin G [4]. While gastrodin and parishin have been the subject of research, there have however been no pharmacokinetic studies of parishin and its analogues. Both gastrodin and parishin are potentially active compounds which may have therapeutic effects in vivo. To gain a better understanding of the molecular mechanisms of GE, it is therefore necessary to investigate the pharmacokinetics of many of its components.

Tall Gastrodia capsules are a traditional Chinese medicine composed of the rhizomes of the GE plants. GE is a prominent and effective traditional Chinese herb that has been used as a neuroprotectant [5], anticonvulsant [6], anti-asthma drug [7], anti-inflammatory and analgesic [8-11]. More than sixty compounds been characterized have to date in GE, including some characteristic parishin compounds $[12,13]$. Previous studies have shown that gastrodin is the most active component in GE [14-19]. Parishin as well as parishin B, C, E, and F and some other structural analogs have been identified or isolated in the GE rhizome as potential active components in vivo. Further research is however required to establish the relationship between the main active gastrodin and parishin compounds, and to identify any transformation of the main compounds which may occur in vivo. In the present study, a systematic and reliable pharmacokinetic quantitative method was developed and validated, and a correlation analysis was conducted between the bioactive compounds in beagle dog plasma.

\section{Results and Discussion}

\subsection{Sample Preparation}

SP 2 was used as a solvent to analyze parishin compounds in GE, especially with regards to pure parishin. SP 3 and SP 4 were based on the results from SP 1 and SP 2, which showed that the target compounds were obtained with good separation efficiency. Formic acid (1\%) was added into SP 5-8, considering the improvement of all the detecting compounds. SP 5 , methanol with $1 \%$ formic acid was ultimately chosen as the best-performing extraction solvent due to good analyte separation and high extraction recovery $(>90 \%)$. In the plasma sample extraction recovery was higher than $90 \%$ when using the five-fold volume of extraction solvent. Under these conditions, the interference with endogenous compounds was significantly inhibited.

\subsection{Elution System}

Parishin $B$ and $C$ were well detected in the aqueous phase of the $0.1 \%$ formic acid-water combination, but not in the other two aqueous phases (Figure 1A). The peak area of parishin $\mathrm{E}$ increased significantly in this condition. Because of this, $0.1 \%$ formic acid-water was chosen as the aqueous phase for further analyses.

The different percentages of the initial mobile phase were important to the matrix effects in some of the target compounds and also played a substantial role in improving the sensitivity, making optimization of the initial mobile phase crucial. As the present study used gastrodin as the water-soluble compound due to its ready inhibition by the endogenous protein, the initial mobile phase was the key condition needing to be optimized. Accordingly, the initial mobile phase was preferable in the following course of optimization. In our study, there was a positive correlation between the sensitivity of gastrodin and the percentage of aqueous phase in the initial mobile phase. Finally, 2\% acetonitrile-water was chosen as the optimal initial mobile phase condition (Figure 1B). However, the high acidity of this phase indicated that acidity may be an important factor affecting 
the resolution, which may in turn affect recovery. In the present study, all candidates were tested with different acid percentages. The findings showed that $0.4 \%$ formic acid was superior to the other candidates (Figure 1C). The data (peak area) were presented in Supplementary materials (Table S1).

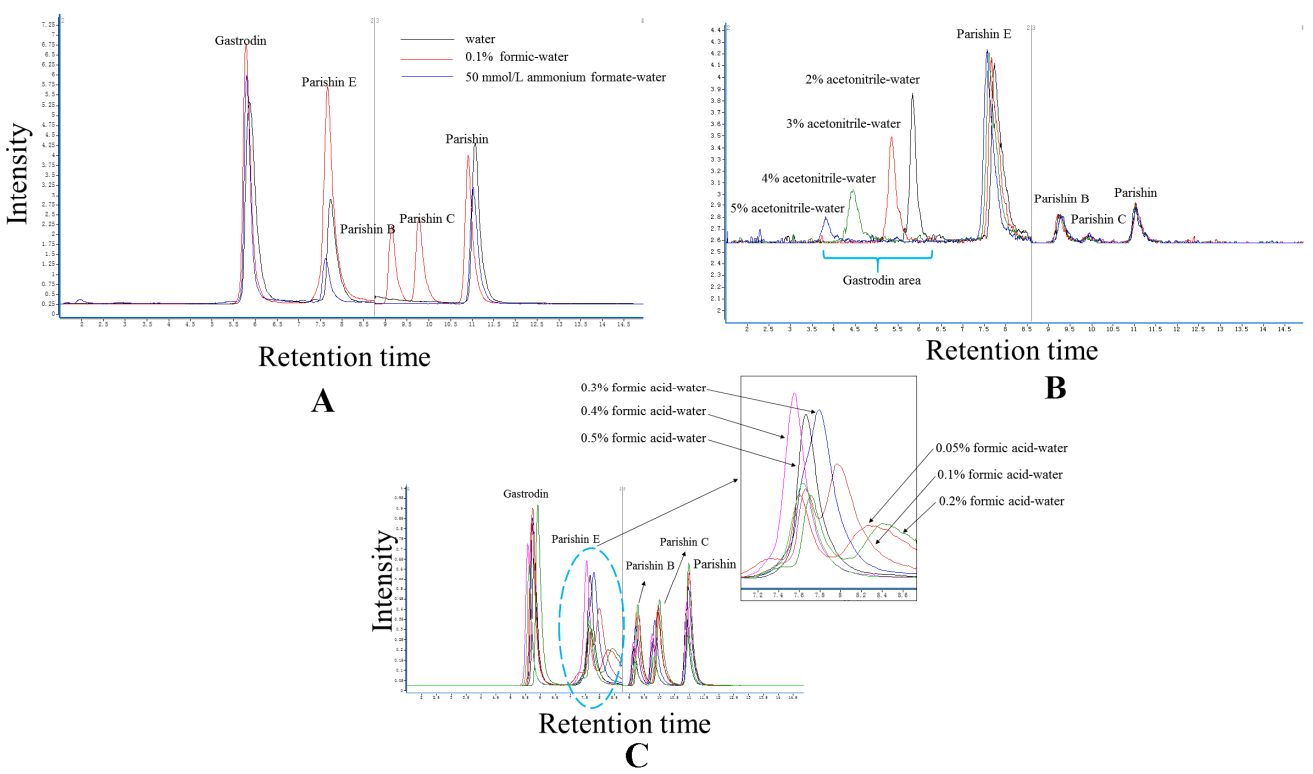

Figure 1. Optimization of elution system. Comparison was presented on the base of total ions mass spectrograms. (A) Type of aqueous phase; (B) Percentage of initial mobile phase; (C) Acidity in aqueous phase.

\subsection{Obtaining Compounds in Plasma Using LC-MS/MS}

Mass ion source parameters were also optimized to maximize sensitivity. All measurements were performed in negative ion mode. This experiment was carried out using automatic injection of the mixture that contained the test compounds in $10 \mu \mathrm{L}$ volumes. The optimization of drying gas temperature and flow rates, sheath gas temperature and flow rates, and nozzle voltage were chosen empirically (Table 1 and Figure S1).

Table 1. Six different ion source parameter combinations.

\begin{tabular}{ccccccc}
\hline & Condition 1 & Condition 2 & Condition 3 & Condition 4 & Condition 5 & Condition 6 \\
\hline $\begin{array}{c}\text { Drying gas } \\
\begin{array}{c}\text { Temperature }\left({ }^{\circ} \mathrm{C}\right) \\
\text { Drying gas flow }\end{array}\end{array}$ & 300 & 300 & 300 & 300 & 300 & 300 \\
$\begin{array}{r}\text { rates (L/min) } \\
\quad \text { Sheath gas }\end{array}$ & 5 & 8 & 8 & 8 & 8 & 8 \\
temperature $\left({ }^{\circ} \mathrm{C}\right)$ & 250 & 400 & 300 & 300 & 350 & 350 \\
$\quad \begin{array}{c}\text { Sheath gas flow } \\
\text { rates }(\mathrm{L} / \mathrm{min})\end{array}$ & 9 & 12 & 12 & 12 & 12 & 12 \\
Nozzle voltage $(\mathrm{kV})$ & 0.5 & 1.0 & 1.0 & 0.5 & 0.5 & 1.0 \\
\hline
\end{tabular}

\subsection{Method Validation}

\subsubsection{Separation, Selectivity, and Sensitivity}

After conducting numerous pre-experiments to establish optimization of bio-sample pretreatments, a well optimized LC-MS/MS method was established that resulted in good peak shape with baseline separation for all analytes with baseline separation from the endogenous plasma components. The method obtained high selectivity with the mass transitions of gastrodin, parishin, parishin B, parishin C, parishin E and bergenin. All analytes were monitored in negative mode. 


\subsubsection{Recovery and Matrix Effect}

The extraction efficiency for gastrodin, parishin, and parishin B, C and E in beagle dog plasma was investigated by measuring the recovery. According to the sample concentration, three concentrations (high, medium and low) of standard solutions were added into known amounts of the sample solution. These were then extracted and subjected to quantitative analysis as described above. Each standard was tested in triplicate at each concentration. Extraction recovery of all analytes ranged from $95.3 \%$ to $101 \%$, indicating that the extraction method had close to optimal accuracy. In addition, the relative standard deviation (RSD) values for all analytes $(<6.93 \%)$ indicated good reproducibility. Matrix effects of all analytes ranged from $96.6 \%$ to $107 \%(n=6)$. The IS value at a concentration level of $0.09408 \mu \mathrm{g} / \mathrm{mL}$ was $102 \pm 2.36(n=6)$. The results showed that the matrix effect on the analysis of the five analytes and the IS in beagle dog plasma was negligible. Results are shown in Table 2.

Table 2. Recovery and matrix effect of gastrodin, parishin, parishin $B$, parishin $C$ and parishin $E$ in beagle dog plasma $(n=6)$.

\begin{tabular}{cccccc}
\hline \multicolumn{2}{c}{ Spiked Conc. (ng/mL) } & Recovery (\%) & RSD (\%) & Matrix Effect (\%) & RSD (\%) \\
\hline \multirow{3}{*}{ Gastrodin } & 3620 & 98.9 & 0.84 & 101 & 2.89 \\
& 723 & 99.5 & 0.81 & 107 & 1.90 \\
& 144 & 98.1 & 1.46 & 99.9 & 4.45 \\
\hline \multirow{3}{*}{ Parishin } & 840 & 98.4 & 2.83 & 103 & 1.91 \\
& 168 & 101 & 2.43 & 107 & 3.82 \\
& 33.6 & 97.0 & 7.65 & 102 & 8.02 \\
\hline \multirow{3}{*}{ Parishin B } & 1040 & 95.3 & 3.08 & 103 & 2.24 \\
& 208 & 95.3 & 4.81 & 96.6 & 2.00 \\
& 41.6 & 98.5 & 5.19 & 103 & 4.64 \\
\hline \multirow{3}{*}{ Parishin C } & 200 & 98.9 & 2.09 & 101 & 4.72 \\
& 40 & 96.5 & 5.55 & 100 & 6.35 \\
& 8 & 97.5 & 6.93 & 100 & 10.8 \\
\hline \multirow{3}{*}{ Parishin E } & 306 & 96.3 & 2.33 & 99.8 & 1.87 \\
& 12.2 & 98.5 & 2.14 & 101 & 1.56 \\
\hline
\end{tabular}

\subsubsection{Precision and Accuracy}

Six samples were extracted and analyzed on three consecutive days to determine the inter-day precision, while six samples were extracted and evaluated on the same day to determine the intra-day precision. Intra- and inter-day precision were assessed using the RSD values of all compounds. All values were below $4.50 \%$, and accuracy deviation was within $98.2 \pm 6.0 \%$ of the actual values at each QC level. These results suggest that the accuracy and precision in the present assay were acceptable for analysis.

\subsubsection{Stability}

Stability under different storage conditions was assessed for all analytes. Analytes in beagle dog plasma were stable at $-20{ }^{\circ} \mathrm{C}$ for 30 days for three freeze-thaw cycles (\%RE, $\left.\pm 5 \%\right)$ and at room temperature $(\% \mathrm{RE}, \pm 15 \%)$. For reconstituted solutions, the stability of all analytes showed no significant degradation when stored in the autosampler for $48 \mathrm{~h}$ (\%RE, $\pm 6 \%$ ).

\subsection{Linearity of Calibration Curves and LLOQs}

Linear regression modeling of the ratio of analytes to the internal standard $(y)$ and plasma concentration $(x)$ for gastrodin yielded the equation $y=3.1278 x+139.87(1.32-4800 \mathrm{ng} / \mathrm{mL}, 0.9990)$; for parishin, $y=1.6481 x-18.496(18-1800 \mathrm{ng} / \mathrm{mL}, 0.9987)$; for parishin $\mathrm{B}, y=1.3725 x-14.674$ (18-2000 ng/mL, 0.9998); for parishin C, $y=1.8269 x-8.9595$ (6-500 ng/mL, 0.9987); and for parishin $\mathrm{E}, y=22.974 x-73.557(4-400 \mathrm{ng} / \mathrm{mL}, 0.9997)$. The correlation coefficient of all analytes was found to 
be $>0.990$, indicating good linearity. LLODs for the above compounds were $0.35,0.83,1.46,0.97$ and $0.067 \mathrm{ng} / \mathrm{mL}$, respectively. These were obtained under conditions where precision and accuracy were below $20 \%$ and the signal-to-noise ratio was 10 .

\subsection{Application to Pharmacokinetic Studies}

After full validation, the LC-ESI-MS/MS method was used to analyze large batches of beagle dog plasma. In the pharmacokinetic study, we successfully and effectively investigated gastrodin, parishin and parishin B, C and E from intragastrically administered tall Gastrodia capsules at low $(0.125 \mathrm{~g} / \mathrm{kg})$, medium $(0.25 \mathrm{~g} / \mathrm{kg})$ and high doses $(0.5 \mathrm{~g} / \mathrm{kg})$. The corresponding pharmacokinetic parameters were generated by fitting plasma concentration profiles to a non-compartmental model. These are summarized in Table 3, expressed as the mean \pm SD $(n=5)$. The mean plasma concentration-time curves $(n=5)$ of five analytes are shown in Figure 2.

Table 3. The mean plasma pharmacokinetic parameters under low, medium and high doses of the five analytes $(n=5)$.

\begin{tabular}{|c|c|c|c|c|c|}
\hline Compounds & Parameter & Unit & Low Dose & Medium Dose & High Dose \\
\hline \multirow[t]{8}{*}{ Gastrodin } & $\mathrm{AUC}_{(0-\mathrm{t})}$ & $\mathrm{ng} / \mathrm{mL} \cdot \mathrm{h}$ & $4890 \pm 2060$ & $8830 \pm 1860$ & $(17.0 \pm 4.24) \times 10^{3}$ \\
\hline & $\mathrm{AUC}_{(0-\infty)}$ & $\mathrm{ng} / \mathrm{mL} \cdot \mathrm{h}$ & $5080 \pm 2110$ & $9170 \pm 1790$ & $(17.6 \pm 4.71) \times 10^{3}$ \\
\hline & $\operatorname{MRT}_{(0-t)}$ & $\mathrm{h}$ & $2.98 \pm 1.01$ & $3.46 \pm 0.29$ & $3.54 \pm 0.69$ \\
\hline & $\operatorname{MRT}_{(0-\infty)}$ & $\mathrm{h}$ & $3.33 \pm 1.09$ & $3.80 \pm 0.28$ & $3.93 \pm 0.99$ \\
\hline & $t_{1 / 2}$ & $\mathrm{~h}$ & $1.86 \pm 0.67$ & $1.90 \pm 0.26$ & $2.09 \pm 0.68$ \\
\hline & CL & $\mathrm{L} / \mathrm{h} / \mathrm{kg}$ & $0.21 \pm 0.13$ & $0.20 \pm 0.04$ & $0.21 \pm 0.05$ \\
\hline & $\mathrm{T}_{\max }$ & $\mathrm{h}$ & $1.10 \pm 0.52$ & $1.75 \pm 0.90$ & $2.00 \pm 0.71$ \\
\hline & $\mathrm{C}_{\max }$ & $\mathrm{ng} / \mathrm{mL}$ & $1200 \pm 120$ & $2050 \pm 495$ & $3760 \pm 778$ \\
\hline \multirow[t]{8}{*}{ Parishin } & $\mathrm{AUC}_{(0-\mathrm{t})}$ & $\mathrm{ng} / \mathrm{mL} \cdot \mathrm{h}$ & $868 \pm 314$ & $1500 \pm 836$ & $3270 \pm 1370$ \\
\hline & $\mathrm{AUC}_{(0-\infty)}$ & $\mathrm{ng} / \mathrm{mL} \cdot \mathrm{h}$ & $899 \pm 299$ & $1520 \pm 832$ & $3280 \pm 1370$ \\
\hline & $\operatorname{MRT}_{(0-t)}$ & $\mathrm{h}$ & $1.80 \pm 0.42$ & $2.49 \pm 0.40$ & $2.48 \pm 0.36$ \\
\hline & $\operatorname{MRT}_{(0-\infty)}$ & $\mathrm{h}$ & $2.00 \pm 0.30$ & $2.65 \pm 0.44$ & $2.50 \pm 0.35$ \\
\hline & $t_{1 / 2}$ & $\mathrm{~h}$ & $1.15 \pm 0.18$ & $1.38 \pm 0.34$ & $1.05 \pm 0.16$ \\
\hline & CL & $\mathrm{L} / \mathrm{h} / \mathrm{kg}$ & $2.69 \pm 1.01$ & $3.56 \pm 1.75$ & $3.18 \pm 1.65$ \\
\hline & $\mathrm{T}_{\max }$ & h & $1.00 \pm 0.56$ & $1.55 \pm 0.62$ & $1.45 \pm 0.76$ \\
\hline & $\mathrm{C}_{\max }$ & $\mathrm{ng} / \mathrm{mL}$ & $388 \pm 116$ & $462 \pm 230$ & $969 \pm 292$ \\
\hline \multirow[t]{8}{*}{ Parishin B } & $\mathrm{AUC}_{(0-\mathrm{t})}$ & $\mathrm{ng} / \mathrm{mL} \cdot \mathrm{h}$ & $1550 \pm 530$ & $2700 \pm 1130$ & $4700 \pm 2330$ \\
\hline & $\mathrm{AUC}_{(0-\infty)}$ & $\mathrm{ng} / \mathrm{mL} \cdot \mathrm{h}$ & $1570 \pm 533$ & $2720 \pm 1140$ & $4720 \pm 2330$ \\
\hline & $\operatorname{MRT}_{(0-t)}$ & $\mathrm{h}$ & $2.25 \pm 0.26$ & $2.78 \pm 0.42$ & $2.76 \pm 0.44$ \\
\hline & $\operatorname{MRT}_{(0-\infty)}$ & $\mathrm{h}$ & $2.32 \pm 0.25$ & $2.84 \pm 0.44$ & $2.80 \pm 0.42$ \\
\hline & $t_{1 / 2}$ & $\mathrm{~h}$ & $1.08 \pm 0.11$ & $1.18 \pm 0.29$ & $1.17 \pm 0.22$ \\
\hline & CL & $\mathrm{L} / \mathrm{h} / \mathrm{kg}$ & $0.72 \pm 0.39$ & $0.78 \pm 0.23$ & $1.11 \pm 0.84$ \\
\hline & $\mathrm{T}_{\max }$ & $\mathrm{h}$ & $1.25 \pm 0.69$ & $1.80 \pm 0.45$ & $2.00 \pm 0.71$ \\
\hline & $\mathrm{C}_{\max }$ & $\mathrm{ng} / \mathrm{mL}$ & $526 \pm 143$ & $746 \pm 320$ & $1220 \pm 562$ \\
\hline \multirow[t]{8}{*}{ Parishin C } & $\mathrm{AUC}_{(0-\mathrm{t})}$ & $\mathrm{ng} / \mathrm{mL} \cdot \mathrm{h}$ & $349 \pm 100$ & $567 \pm 198$ & $1090 \pm 523$ \\
\hline & $\mathrm{AUC}_{(0-\infty)}$ & $\mathrm{ng} / \mathrm{mL} \cdot \mathrm{h}$ & $359 \pm 99.3$ & $578 \pm 200$ & $1100 \pm 527$ \\
\hline & $\operatorname{MRT}_{(0-t)}$ & $\mathrm{h}$ & $2.47 \pm 0.28$ & $3.14 \pm 0.54$ & $3.18 \pm 0.69$ \\
\hline & $\operatorname{MRT}_{(0-\infty)}$ & $\mathrm{h}$ & $2.70 \pm 0.28$ & $3.31 \pm 0.59$ & $3.25 \pm 0.72$ \\
\hline & $t_{1 / 2}$ & $\mathrm{~h}$ & $1.37 \pm 0.18$ & $1.56 \pm 0.41$ & $1.29 \pm 0.36$ \\
\hline & CL & $\mathrm{L} / \mathrm{h} / \mathrm{kg}$ & $0.72 \pm 0.23$ & $0.91 \pm 0.23$ & $1.26 \pm 1.05$ \\
\hline & $\mathrm{T}_{\max }$ & $\mathrm{h}$ & $1.35 \pm 0.60$ & $2.00 \pm 0.71$ & $2.40 \pm 0.55$ \\
\hline & $\mathrm{C}_{\max }$ & $\mathrm{ng} / \mathrm{mL}$ & $107 \pm 29.4$ & $144 \pm 55.1$ & $243 \pm 99.6$ \\
\hline \multirow[t]{8}{*}{ Parishin E } & $\mathrm{AUC}_{(0-\mathrm{t})}$ & $\mathrm{ng} / \mathrm{mL} \cdot \mathrm{h}$ & $545 \pm 188$ & $865 \pm 257$ & $1620 \pm 76$ \\
\hline & $\operatorname{AUC}_{(0-\infty)}$ & $\mathrm{ng} / \mathrm{mL} \cdot \mathrm{h}$ & $553 \pm 190$ & $868 \pm 254$ & $1630 \pm 759$ \\
\hline & $\operatorname{MRT}_{(0-t)}$ & $\mathrm{h}$ & $3.86 \pm 1.05$ & $3.74 \pm 0.66$ & $4.36 \pm 0.59$ \\
\hline & $\operatorname{MRT}_{(0-\infty)}$ & $\mathrm{h}$ & $4.19 \pm 1.36$ & $3.79 \pm 0.60$ & $4.52 \pm 0.66$ \\
\hline & $t_{1 / 2}$ & $\mathrm{~h}$ & $3.09 \pm 2.03$ & $1.72 \pm 0.13$ & $3.00 \pm 1.71$ \\
\hline & $\mathrm{CL}$ & $\mathrm{L} / \mathrm{h} / \mathrm{kg}$ & $1.33 \pm 0.82$ & $1.50 \pm 0.35$ & $2.01 \pm 1.44$ \\
\hline & $\mathrm{T}_{\max }$ & $\mathrm{h}$ & $1.35 \pm 0.60$ & $2.00 \pm 0.71$ & $1.80 \pm 0.45$ \\
\hline & $\mathrm{C}_{\max }$ & $\mathrm{ng} / \mathrm{mL}$ & $136 \pm 35.3$ & $192 \pm 57.0$ & $312 \pm 138$ \\
\hline
\end{tabular}



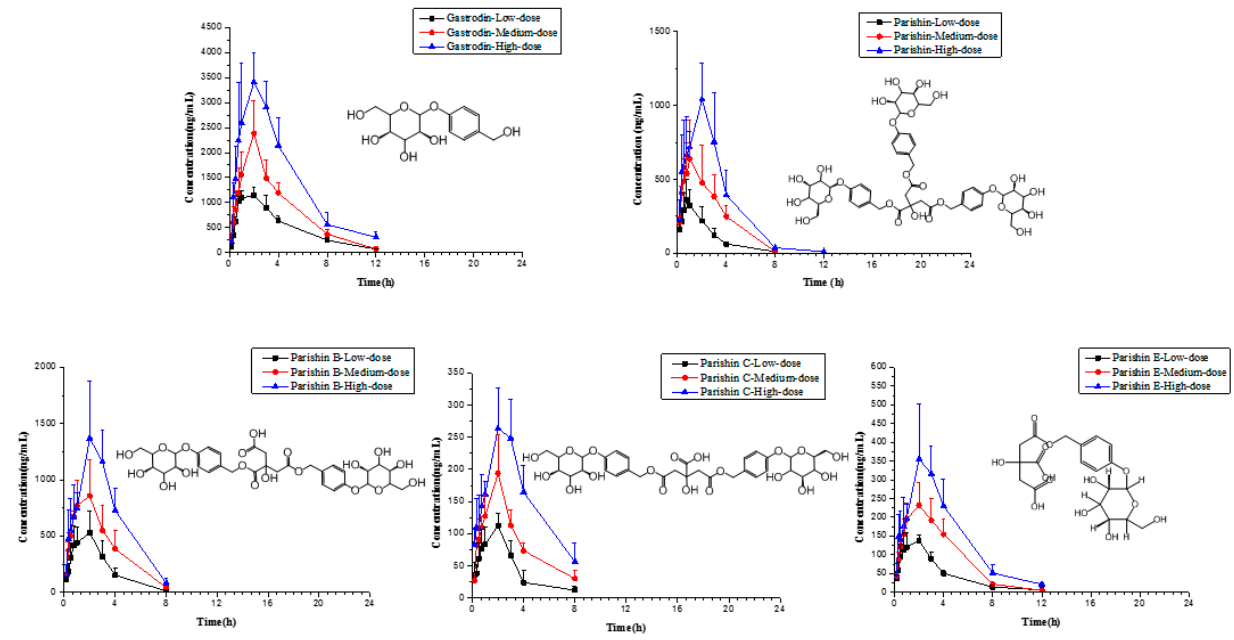

Figure 2. Mean plasma concentration-time profile of five compounds in beagle dog after intragastric administration of low dose, medium dose and high dose tall Gastrodia capsules $(n=5)$.

Gastrodin was detected in beagle dog plasma 10 min after intragastric administration of capsules at all three dosage levels. For low, medium and high doses, retention time of the maximum plasma concentration $\left(\mathrm{T}_{\max }\right)$ was reached at $1.10,1.75$ and $2.00 \mathrm{~h}$, respectively. These results were similar to the findings of earlier studies $[1,20]$. However, $\mathrm{T}_{\max }$ in the present study was roughly double that found in rats [21]. This shows that the onset time of effects from gastrodin in beagle dogs is longer than that found in rats. In addition, $\mathrm{T}_{\max }$ for the high dose condition was twice long as in the low dose condition, indicating that the amount of gastrodin in vivo may influence the time of maximum plasma concentration. The area under plasma concentration-time curve from 0 to infinity $\left(\mathrm{AUC}_{0-\mathrm{t}}\right)$ of gastrodin was $4890 \pm 2060,8830 \pm 1860$ and $(17.0 \pm 4.24) \times 10^{3} \mathrm{ng} / \mathrm{mL} \cdot \mathrm{h}$ for low, medium and high doses, respectively, and area under plasma concentration-time curve from 0 to infinity $\left(\mathrm{AUC}_{0-\infty}\right)$ was $5080 \pm 2110,9170 \pm 1790$ and $(17.6 \pm 4.71) \times 10^{3} \mathrm{ng} / \mathrm{mL} \cdot \mathrm{h}$. Comprehensive area under plasma concentration-time curve (AUC) analysis of all five analytes showed that gastrodin was the constituent with the highest concentration in plasma. The concentration of parishin B was one-third the concentration of gastrodin, and the other three compounds had lower concentrations.

Several previous studies of GE have focused primarily on gastrodin [22-24]. However, both parishin and parishin B are also potentially active compounds based on their high concentrations in vivo and should therefore be investigated more deeply at a pharmacological level. At the same time Jiang et al. reported [4] that gastrodin can be detected in heart, liver and other tissues, which could be expanded the pharmacodynamics of gastrodin study. Mean plasma clearance $(\mathrm{CL})$ for gastrodin, parishin, and parishin $\mathrm{B}, \mathrm{C}$ and $\mathrm{E}$ was $0.2,3.14,0.87,0.96$ and $1.61 \mathrm{~L} / \mathrm{h} / \mathrm{kg}$, respectively, showing that gastrodin was eliminated more rapidly than any of the other compounds in beagle dogs. The maximum plasma concentration $\left(C_{\max }\right)$ of gastrodin was $1200 \pm 120,2050 \pm 495$ and $3760 \pm 778 \mathrm{ng} / \mathrm{mL}$ in the low, medium, and high intragastric administration levels, respectively. Besides, for the three dosage levels, mean residence time from 0 to $24 \mathrm{~h}\left(\mathrm{MRT}_{0-\mathrm{t}}\right)$ was $2.98 \pm 1.01$, $3.46 \pm 0.29$ and $3.54 \pm 0.69 \mathrm{~h}$; mean residence time from 0 to infinity $\left(\mathrm{MRT}_{0-\infty}\right)$ was $3.33 \pm 1.09$, $3.80 \pm 0.28$ and $3.93 \pm 0.99 \mathrm{~h}$; and terminal elimination half-life $\left(\mathrm{t}_{1 / 2}\right)$ was $1.86 \pm 0.67,1.90 \pm 0.26$ and $2.09 \pm 0.68 \mathrm{~h}$.

\subsection{Correlation Analysis}

Resultant pharmacokinetic data were then used to assess correlations between the four parishin compounds and gastrodin in vivo. Correlation analysis was conducted using SPSS 16.0 software. Pearson's correlation coefficient was used to investigate correlations among the five compounds. Correlation coefficients were low among the five compounds at all doses (mean: 0.54). 
However, stronger correlations were found between parishin, parishin $B$, parishin $C$ and parishin $E$. Parishin and parishin B, parishin and parishin $C$, and parishin and parishin E comprised Group 1 (mean: 0.76). Parishin B and parishin E, and parishin C and parishin E comprised Group 2 (mean: 0.95). These correlation coefficients (Figure 3) suggest that gastrodin and parishin compounds can be biotransformed into each other at different rates in vivo. This finding is consistent with previous reports of biotransformation between parishins and gastrodin [4], furthermore, our detected compounds were also reported by Tang et al. [25] using the Q-TOF-MS/MS.

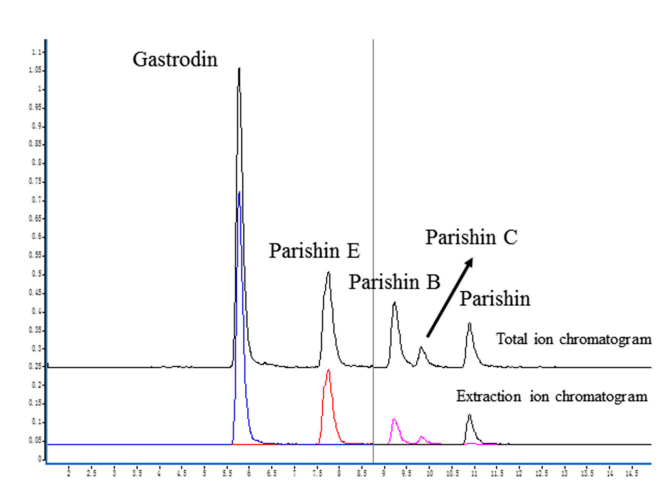

A

\begin{tabular}{|c|c|c|c|c|c|}
\hline & Gastrodin & Parishin E & Parishin B & Parishin C & Parishin \\
\hline Gastrodin & 1 & 0.54 & 0.55 & 0.58 & 0.57 \\
\hline Parishin E & & 1 & $0.99^{\star \star}$ & $0.93^{\star \star}$ & $0.77^{\star \star}$ \\
\hline Parishin B & High & & 1 & $0.93^{\star \star}$ & $0.83^{\star \star \star}$ \\
\hline Parishin C & & & & 1 & $0.81^{\star \star}$ \\
\hline $\begin{array}{l}\text { Parishin } \\
\text { Gatrodin }\end{array}$ & & & & & 1 \\
\hline Gastrodin & 1 & 0.43 & 0.39 & 0.42 & 0.19 \\
\hline Parishin E & & 1 & $0.97^{\star \star}$ & $0.91^{\star \star}$ & $0.75^{\star \star}$ \\
\hline Parishin B & \multirow{3}{*}{\multicolumn{2}{|c|}{ Medium }} & 1 & $0.922^{\star \star}$ & $0.83^{\star \star \star}$ \\
\hline Parishin C & & & & 1 & $0.73^{\star \star}$ \\
\hline Parishin & & & & & 1 \\
\hline Gastrodin & 1 & 0.79 & 0.83 & 0.79 & 0.44 \\
\hline Parishin E & \multirow{4}{*}{ Low } & 1 & $0.98^{\star \star}$ & $0.94^{\star \star}$ & $0.75^{\star \star}$ \\
\hline Parishin B & & & 1 & $0.96^{\star \star}$ & $0.75^{\star \star}$ \\
\hline Parishin C & & & & 1 & $0.66^{\star \star}$ \\
\hline Parishin & & & & & 1 \\
\hline
\end{tabular}

B

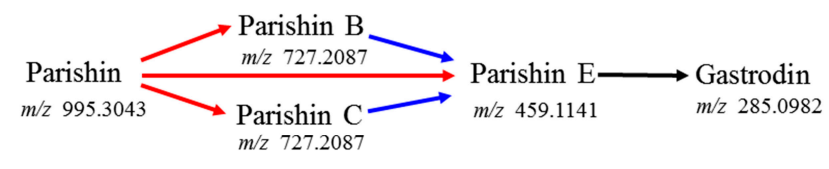

C

Figure 3. (A) The total ion mass spectrograms and extraction ion mass spectrogram of the potential compounds in beagle plasma after administration of tall Gastrodia capsules; (B) Correlation coefficients of five compounds ( ${ }^{* *}$ represents $\left.p<0.01\right)$; (C) Putative pathway from parishin to gastrodin in vivo.

\section{Experimental}

\subsection{Materials and Reagents}

Reference compounds of parishin and parishin B, C and E were purchased from Jiangxi Herbfine Hi-Tech (Nanchang, Jiangxi, China). Bergenin as internal standard (IS) and gastrodin were purchased from the National Institute for the Control of Biological and Pharmaceutical Drugs (Beijing, China). The purity of reference substances was above 98\%. Tall Gastrodia capsules were kindly supplied by Guizhou Eakan Pharmaceutical (Guiyang, China). Methanol and acetonitrile of MS grade were acquired from Sigma-Aldrich (St. Louis, MO, USA) and formic acid used for HPLC-MS analysis from Anaqua Chemicals Supply (Houston, TX, USA). Ultrapure water was produced using a Milli-Q water purification system (Millipore, Bedford, MA, USA). All other reagents used in the present study were analytical grade.

\subsection{Instruments and Analytical Conditions}

Quantitative analysis was conducted on a 1290 UPLC-photodiode array 6460 triple quadrupole mass spectrometry system (LC-MS/MS, Agilent Technologies, Palo Alto, CA, USA). Analysis was carried out on. Separation of analytes was performed on a reversed phase Agilent Zorbax XDB-C 18 HPLC column $(2.1 \mathrm{~mm} \times 50 \mathrm{~mm}$, particle size $3.5 \mu \mathrm{m})$ with the column oven set to $30{ }^{\circ} \mathrm{C}$. The elution gradient was carried out with a binary solvent system consisting of an aqueous solution of $0.4 \% v / v$ formic acid in water $(\mathrm{A})$ and acetonitrile $(\mathrm{B})$ at a flow rate of $0.15 \mathrm{~mL} / \mathrm{min}$. Optimized separation was obtained according to a linear gradient (0-2 $\mathrm{min}, 2 \% \mathrm{~B} ; 2-4 \mathrm{~min}, 2-12 \% \mathrm{~B} ; 4-8 \mathrm{~min}, 12-20 \%$ B; 8-14 min, 20-28\% B; 12-15 min, 28-90\% B). MS/MS conditions were as follows: negative ion 
mode, drying gas $\left(\mathrm{N}_{2}\right)$ flow rate $8.0 \mathrm{~L} / \mathrm{min}$, drying gas temperature $300{ }^{\circ} \mathrm{C}$, declustering potential $150 \mathrm{~V}$, nebulizer pressure 45 psi, ESI Vcap $3500 \mathrm{~V}$, sheath gas temperature $300{ }^{\circ} \mathrm{C}$, sheath gas flow $12 \mathrm{~L} / \mathrm{min}$, nozzle voltage $500 \mathrm{~V}$. The mass transition of gastrodin $(\mathrm{m} / z 331.1 \rightarrow 123.0, \mathrm{CE}: 3 \mathrm{eV})$, parishin $(m / z$ 995.4 $\rightarrow 727.0, \mathrm{CE}: 42 \mathrm{eV})$, parishin B $(m / z 727.3 \rightarrow 423.0, \mathrm{CE}: 20 \mathrm{eV})$, parishin $\mathrm{C}(\mathrm{m} / z$ 727.3 $\rightarrow 423.1$, $\mathrm{CE}: 20 \mathrm{eV})$, parishin $\mathrm{E}(m / z 459.3 \rightarrow 110.9, \mathrm{CE}: 19 \mathrm{eV})$ and bergenin $(m / z$ 327.0 $\rightarrow 234.0, \mathrm{CE}: 4 \mathrm{eV})$.

\subsection{Animal Experiments}

Adult male Sprague-Dawley (SD) beagle dogs (12 kg $\pm 0.020 \mathrm{~kg}$ ) were purchased from Beijing Shahe Bio-Technology (Beijing, China; certificate No. SCXK2014-0007). Before drug administration, the dogs were fasted for $12 \mathrm{~h}$ with free access to water. They were maintained on a $12 \mathrm{~h}$ light-dark cycle (light on from 6:00 to $18: 00 \mathrm{~h}$ ) at ambient temperature $\left(22-24^{\circ} \mathrm{C}\right)$ with $60 \%$ relative humidity. The animals were observed twice daily and body weights were recorded once per day to assess their general health. All experimental protocols on animals were carried out according to the Guidelines of the Committee on the Care and Use of Laboratory Animals of China.

\subsection{Drug Administration and Blood Sampling}

Fifteen beagle dogs were randomly divided into three groups to receive an intragastric dose of tall Gastrodia capsules $(0.125,0.25$ and $0.5 \mathrm{~g} / \mathrm{kg})$. Capsules were ultrasonically dissolved in pure water and suspended at concentrations of 5, 10 and $20 \mathrm{mg} / \mathrm{mL}$ for intragastric administration. The concentration of gastrodin, parishin, parishin B, parishin C are measured at $3.29 \mathrm{mg} / \mathrm{g}, 4.482 \mathrm{mg} / \mathrm{g}, 2.258 \mathrm{mg} / \mathrm{g}$, $0.69 \mathrm{mg} / \mathrm{g}$, respectively. The HPLC quantitative analysis was based on the previous report with some modification [26]. After administration, serial blood samples (about $2.00 \mathrm{~mL}$ ) were collected from the foreleg vein (the ophthalmic artery plexus) into heparin-containing capillary tubes at 10, 20, 30, 45, 60, 120, 180, 240, 480, 720 and $1440 \mathrm{~min}$. Blood samples were promptly centrifuged at $5000 \mathrm{rpm}$ for $5 \mathrm{~min}$, and the plasma was separated and stored at $-20^{\circ} \mathrm{C}$ until analysis.

\subsection{Experimental Condition Optimization}

The aims of the optimization experiment for LC-MS/MS were not only to obtain higher analysis efficiency with good recovery, but also to remove matrix interference. The optimization of LC-MS/MS conditions for all tested compounds was achieved in vivo after intragastric administration of tall Gastrodia capsules. Different candidate systems of sample preparation (SP)/elution system (ES) combinations were used for optimization.

Based on analysis of relevant previous research [1-3], eight candidate sample preparation systems with different organic solvents were tested to optimize the five quantitative compounds and bergenin in plasma samples (hereafter SP 1-8). Sample preparation procedures were classified into four groups. For Group 1 (SP 1, SP 5) methanol was used as the extraction solvent; for Group 2 (SP 2, SP 6), acetonitrile; for Group 3 (SP 3, SP 7), a 1:1 mixture of methanol and ethanol; for Group 4 (SP 4, SP 8), a 1:1:1 mixture of methanol, acetonitrile and acetone. In addition, SP 5-8 were combined with $1 \%$ formic acid, whereas SP 1-4 were not.

The elution systems included an aqueous phase and an initial mobile phase. The acidity of the mobile phase was also optimized. The three candidate elution systems (ES) consisted of water, $0.1 \%$ formic acid-water and $50 \mathrm{mmol} / \mathrm{L}$ ammonium formate-water, which were commonly used in previous studies but optimized here.

The acidity of the mobile phase was also an important factor for the analysis of gastrodin, parishin, and parishin B, C and E. Different levels of acidity were achieved through the addition of 0.05, 0.1, 0.2, $0.3,0.4$, or $0.5 \%$ formic acid into the water phase, and were compared when assessing the results.

\subsection{Plasma Sample Preparation}

Plasma samples were thawed to $4{ }^{\circ} \mathrm{C}$ before processing. An aliquot of $200 \mu \mathrm{L}$ of the plasma sample was transferred into an Eppendorf tube, and $1000 \mu \mathrm{L}$ of ice-cold $1 \%$ formic acid methanol 
containing IS $(10 \mu \mathrm{L}, 2.0 \mu \mathrm{g} / \mathrm{mL})$ was added to precipitate the plasma proteins. Samples were then vortexed for $3 \mathrm{~min}$ and centrifuged at 12,000 rpm for $10 \mathrm{~min}$. The supernatant was transferred and evaporated to dryness under a gentle stream of nitrogen at $40{ }^{\circ} \mathrm{C}$. The residue was reconstituted in $200 \mu \mathrm{L}$ of $5.0 \%$ methanol-water, and a $10 \mu \mathrm{L}$ aliquot was injected for analysis.

\subsection{Validation of the Method}

Specificity and selectivity were evaluated by analyzing chromatogram comparisons between blank plasma, blank plasma spiked with IS/analytes, and dog plasma samples. Calibration curves were established from peak area ratios (analyte/IS) versus concentrations. QC samples at low, medium and high concentrations were designed to assess the intra- and inter-day precision. In addition, stability (short- and long-term stabilities), extraction recoveries, limit of detection (LOD), limit of quantification (LOQ) and linearity were also investigated.

\subsection{Pharmacokinetic Study and Data Analysis}

The pharmacokinetic parameters were calculated for each subject using the Drug and Statistics (DAS) software package (version 3.0; www.drugchina.net). Parameters consisted of maximum plasma concentration $\left(\mathrm{C}_{\max }\right)$, corresponding time $\left(\mathrm{T}_{\max }\right)$, area under plasma concentration-time curve from 0 to $24 \mathrm{~h}\left(\mathrm{AUC}_{0-\mathrm{t}}\right)$, area under plasma concentration-time curve from 0 to infinity $\left(\mathrm{AUC}_{0-\infty}\right)$, mean residence time from 0 to $24 \mathrm{~h}\left(\mathrm{MRT}_{0-\mathrm{t}}\right)$, mean residence time from 0 to infinity $\left(\mathrm{MRT}_{0-\infty}\right)$, terminal elimination half-life $\left(t_{1 / 2}\right)$ and plasma clearance $(C L)$. A non-compartmental model was used to calculate the parameters. Results are presented as the mean \pm standard deviation (S.D.).

Correlation analysis was conducted using SPSS 16.0 for Windows (IBM, Armonk, NY, USA). Pearson's correlation coefficient was used to investigate correlations between the five compounds.

\section{Conclusions}

A variety of studies in recent years have investigated different methods to establish the presence of gastrodin in plasma samples. One study found that LLOQs of UV detection reached $100 \mathrm{ng} / \mathrm{mL}$ [21]. Research has also validated the use of fluorescence detection for quantification of parishin and its metabolites in rats at LLOQs of $2.5 \mathrm{ng} / \mathrm{mL}$ [27]. Some studies investigated the comparative pharmacokinetics of parishin, gastrodin, Gastrodia elata extract and Rhizoma Gastrodiae capsules using a LC-MS method [21]. Researchers have investigated the holistic pharmacokinetics of gastrodin injection and Rhizoma Gastrodiae capsules, but did not conduct a detailed analysis. In the present study, a rapid, sensitive and reliable LC-MS/MS method was for the first time developed and validated for the simultaneous determination of five active and potential compounds of tall Gastrodia capsules in beagle dog plasma.

In this present study, a validated LC-MS/MS method was firstly used for simultaneous determination of five bioactive compounds in dog plasma. Correlation analysis method was firstly used to analysis the correlation between gastrodin and parishin compounds. Preliminary experiments allowed optimization to the point was a good separation of two isomers (parishin B and parishin C) was successfully obtained. With this excellent sensitive, accurate and fast bioanalytical method, a good and reliable pharmacokinetic study was established. The results might be helpful for investigating the bioactivity mechanism and clinical application of Tianma.

Supplementary Materials: Supplementary materials are available online. Figure S1: Total ions mass spectrograms obtained from different ion source parameters. Table S1: Intensity of gastrodin, parishin, parishin B, parishin C and parishin $\mathrm{E}$ under different experimental condition.

Acknowledgments: The authors are grateful for financial support from the Major Scientific and Technological Special Project for "Significant New Drugs Creation" (No. 2014ZX09304307). The funders had no role in study design, data collection and analysis, decision to publish, or preparation of the manuscript. 
Author Contributions: An Liu and Yuesheng Wang conceived and designed the experiments; Junqiu Liu performed the experiments and contributed to the acquisition of data; Junqiu Liu and Sha Chen analyzed the data; Junqiu Liu and Sha Chen wrote the paper; Jun Zhang and Jintang Cheng contributed reagents/materials/analysis tools. All authors have revised the manuscript critically for important intellectual content and given final approval of the version to be published. An Liu is responsible for the integrity of the work as a whole.

Conflicts of Interest: The authors declare no conflict of interest.

\section{References}

1. Jia, Y.; Li, X.; Xie, H.; Shen, J.; Luo, J.; Wang, J.; Wang, K.D.; Liu, Q.; Kong, L. Analysis and pharmacokinetics studies of gastrodin and $p$-hydroxybenzyl alcohol in dogs using ultra fast liquid chromatography-tandem mass spectrometry method. J. Pharm. Biomed. Anal. 2014, 99, 83-88. [CrossRef] [PubMed]

2. Tang, C.L.; Wang, L.; Liu, X.X.; Cheng, M.; Qu, Y.; Xiao, H.B. Comparative pharmacokinetics of gastrodin in rats after intragastric administration of free gastrodin, parishin and Gastrodia elata extract. J. Ethnopharmacol. 2015, 176, 49-54. [CrossRef] [PubMed]

3. Zhao, Y.; Gong, X.J.; Zhou, X.; Kang, Z.J. Relative bioavailability of gastrodin and parishin from extract and powder of Gastrodiae Rhizoma in rat. J. Pharm. Biomed. Anal. 2014, 100, 309-315. [CrossRef] [PubMed]

4. Tang, C.L.; Wang, L.; Li, J.J.; Liu, X.X.; Cheng, M.C.; Xiao, H.B. Analysis of the metabolic profile of parishin by ultra-performance liquid chromatography/quadrupole-time of flight mass spectrometry. Biomed. Chromatogr. 2015, 29, 1913-1920. [CrossRef] [PubMed]

5. Huang, N.K.; Chern, Y.; Fang, J.M.; Lin, C.I.; Chen, W.P.; Lin, Y.L. Neuroprotective principles from Gastrodia elata. J. Nat. Prod. 2007, 70, 571-574. [CrossRef] [PubMed]

6. Hsieh, C.L.; Chiang, S.Y.; Cheng, K.S.; Lin, Y.H.; Tang, N.Y.; Lee, C.J.; Pon, C.Z.; Hsieh, C.T. Anticonvulsive and free radical scavenging activities of Gastrodia elata Bl. in kainic acid-treated rats. Am. J. Chin. Med. 2001, 29, 331-341. [CrossRef] [PubMed]

7. Jang, Y.W.; Lee, J.Y.; Kim, C.J. Anti-asthmatic activity of phenolic compounds from the roots of Gastrodia elata Bl. Int. Immunopharmacol. 2010, 10, 147-154. [CrossRef] [PubMed]

8. Su, S.; Wang, T.; Duan, J.A.; Zhou, W.; Hua, Y.Q.; Tang, Y.P.; Yu, L.; Qian, D.W. Anti-inflammatory and analgesic activity of different extracts of Commiphora myrrha. J. Ethnopharmacol. 2011, 134, 251-258. [CrossRef] [PubMed]

9. Hwang, S.M.; Lee, Y.J.; Kang, D.G.; Lee, H.S. Anti-inflammatory effect of Gastrodia elata rhizome in human umbilical vein endothelial cells. Am. J. Chin. Med. 2009, 37, 395-406. [CrossRef] [PubMed]

10. Ahn, E.K.; Jeon, H.J.; Lim, E.J.; Jung, H.J.; Park, E.H. Anti-inflammatory and anti-angiogenic activities of Gastrodia elata Blume. J. Ethnopharmacol. 2007, 110, 476-482. [CrossRef] [PubMed]

11. Lee, J.Y.; Jang, Y.W.; Kang, H.S.; Moon, H.; Sim, S.S.; Kim, C.J. Anti-inflammatory action of phenolic compounds from Gastrodia elata root. Arch. Pharm. Res. 2006, 29, 849-858. [CrossRef] [PubMed]

12. Li, Z.; Wang, Y.; Ouyang, H.; Lu, Y.; Qiu, Y.; Feng, Y.; Jiang, H.; Zhou, X.; Yang, S. A novel dereplication strategy for the identification of two new trace compounds in the extract of Gastrodia elata using UHPLC/Q-TOF-MS/MS. J. Chromatogr. B 2015, 988, 45-52. [CrossRef] [PubMed]

13. Chen, S.; Liu, J.Q.; Xiao, H.; Zhang, J.; Liu, A. Simultaneous Qualitative assessment and quantitative analysis of metabolites (phenolics, nucleosides and amino acids) from the roots of fresh Gastrodia elata using UPLC-ESI-Triple quadrupole ion MS and ESI-linear ion trap high-resolution MS. PLoS ONE 2016, 11, e0150647. [CrossRef] [PubMed]

14. Yu, S.S.; Zhao, J.; Zheng, W.P.; Zhao, Y. Neuroprotective effect of 4-hydroxybenzyl alcohol against transient focal cerebral ischemia via anti-apoptosis in rats. Brain Res. 2010, 1308, 167-175. [CrossRef] [PubMed]

15. Dai, J.N.; Zong, Y.; Zhong, L.M.; Li, Y.M.; Zhang, W.; Bian, L.G.; Ai, Q.L.; Liu, Y.D.; Sun, J.; Lu, D. Gastrodin inhibits expression of inducible NO synthase, cyclooxygenase-2 and proinflammatory cytokines in cultured LPS-stimulated microglia via MAPK pathways. PLoS ONE 2011, 6, e21891. [CrossRef] [PubMed]

16. Zhu, L.; Guan, H.; Cui, C.; Tian, S.; Yang, D.; Wang, X.; Zhang, S.; Wang, L.; Jiang, H. Gastrodin inhibits cell proliferation in vascular smooth muscle cells and attenuates neointima formation in vivo. Int. J. Mol. Med. 2012, 30, 1034-1040. [CrossRef] [PubMed]

17. Shu, C.; Chen, C.; Zhang, D.P.; Guo, H.; Zhou, H.; Zong, J.; Bian, Z.; Dong, X.; Dai, J.; Zhang, Y.; et al. Gastrodin protects against cardiac hypertrophy and fibrosis. Mol. Cell. Biochem. 2012, 359, 9-16. [CrossRef] [PubMed] 
18. Wang, H.; Zhang, R.; Qiao, Y.; Xue, F.; Nie, H.; Zhang, Z.; Wang, Y.; Peng, Z.; Tan, Q. Gastrodin ameliorates depression-like behaviors and up-regulates proliferation of hippocampal-derived neural stem cells in rats: Involvement of its anti-inflammatory action. Behav. Brain Res. 2014, 266, 153-160. [CrossRef] [PubMed]

19. Zhao, S.; Li, N.; Zhen, Y.; Ge, M.; Li, Y.; Yu, B.; He, H.; Shao, R.G. Protective effect of gastrodin on bile duct ligation-induced hepatic fibrosis in rats. Food Chem. Toxicol. 2015, 86, 202-207. [CrossRef] [PubMed]

20. Jiang, L.; Dai, J.; Huang, Z.; Du, Q.; Lin, J.; Wang, Y. Simultaneous determination of gastrodin and puerarin in rat plasma by HPLC and the application to their interaction on pharmacokinetics. J. Chromatogr. B 2013, 915, 8-12. [CrossRef] [PubMed]

21. Tang, C.L.; Wang, L.; Liu, X.X.; Cheng, M.C.; Xiao, H.B. Pharmacokinetic study of Gastrodia elata in rats. Anal. Bioanal. Chem. 2015, 407, 8903-8910. [CrossRef] [PubMed]

22. Zeng, X.; Zhang, S.; Zhang, L.; Zhang, K.; Zheng, X. A study of the neuroprotective effect of the phenolic glucoside gastrodin during cerebral ischemia in vivo and in vitro. Planta Med. 2006, 72, 1359-1365. [CrossRef] [PubMed]

23. Wang, X.L.; Xing, G.H.; Hong, B.; Li, X.M.; Zou, Y.; Zhang, X.J.; Dong, M.X. Gastrodin prevents motor deficits and oxidative stress in the MPTP mouse model of Parkinson's disease: Involvement of ERK1/2-Nrf2 signaling pathway. Life Sci. 2014, 114, 77-85. [CrossRef] [PubMed]

24. Wang, P.H.; Zhao, L.X.; Wan, J.Y.; Zhang, L.; Mao, X.N.; Long, F.Y.; Zhang, S.; Chen, C.; Du, J.R. Pharmacological characterization of a novel gastrodin derivative as a potential anti-migraine agent. Fitoterapia 2015, 109, 52-57. [CrossRef] [PubMed]

25. Jiang, Z.; Zheng, X.; Gong, X.; Chao, Z.; Xin, Z.; Yang, Z.; Yan, Y. Relative tissue distribution and excretion studies of gastrodin and parishin from powder and extract of Gastrodiae Rhizoma in rat by UHPLC-ESI-MS/MS. Biomed. Chromatogr. 2016. [CrossRef] [PubMed]

26. Liu, Z.; Wang, A.M.; Chi, M.Y.; Wang, Y.L. Quality evaluation of tall Gastrodia capsules by simultaneous determination of the contents of multi-constituents. Chin. Pharm. J. 2012, 5, 380-383.

27. Tang, C.L.; Wang, L.; Cheng, M.C.; Zhang, X.Z.; Liu, X.Y.; Xiao, H.B. Rapid and sensitive analysis of parishin and its metabolites in rat plasma using ultra high performance liquid chromatography-fluorescence detection. J. Chromatogr. B 2014, 973, 104-109. [CrossRef] [PubMed]

Sample Availability: Samples of the compounds gastrodin, parishin, parishin B, parishin C, parishin E and bergenin are available from the authors. 